\title{
NREL's Black Silicon Increases Solar Cell Efficiency by Reducing Reflected Sunlight
}

\author{
An inexpensive, one-step nanocatalytic wet-chemical \\ etch makes high-efficiency solar cells
}

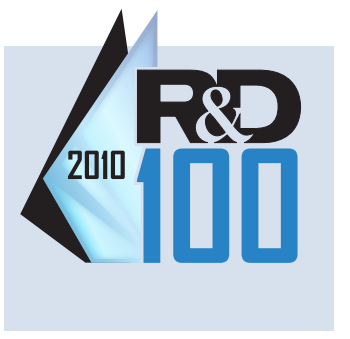

If you want an efficient solar cell, you want the cell to absorb as much of the light shining on it as possible. Less reflection means more light absorption, and hence, higher efficiency and more electricity.

The makers of solar cells have devised ways to boost the amount of light absorbed-including a micrometer-scale textured surface and a thin layer of a material with optical properties different than the underlying solar energy converter. Unfortunately, the equipment and processes for applying these conventional antireflection layers add cost to the solar cell. But even when both techniques are used, cells still only absorb between $93 \%$ and $97 \%$ of the sunlight.

To further reduce reflected sunlight and increase cell efficiency at lower cost, NREL scientists invented the Black Silicon Nanocatalytic Wet-Chemical Etch. This antireflection etch process turns silicon wafers-the most common solar cell material-black because they absorb more than $98 \%$ of the light shining on them. And NREL has validated its black silicon cells at $16.8 \%$ conversion efficiency.

\section{So How Does It Work?}

The NREL team created an inexpensive liquid etch that creates gold nanoparticles that immediately begin to catalyze the etch to produce a nanometerscale porous surface on the cell wafer's surface. The porous features must be much smaller than the wavelength of the incident light; therefore, nanoscale features are needed to suppress reflection of the entire spectrum of sunlight.

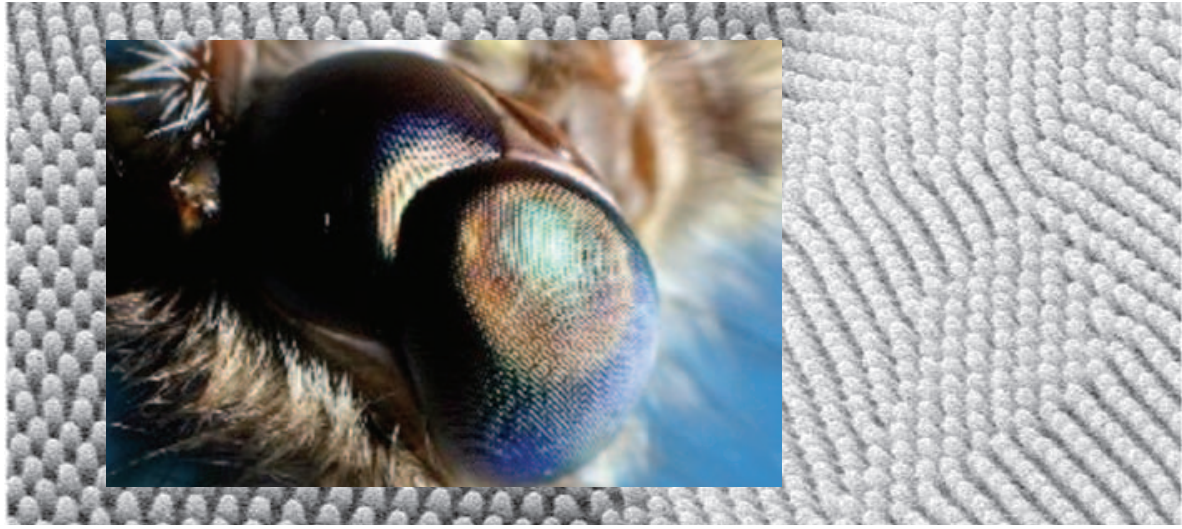

Photo of the entire eye of a moth, overlaying a scanning electron micrograph of a portion of the lens of a moth's eye, where each bump is only several hundred nanometers in diameter. NREL's black silicon etch creates nanostructures akin to those of the moth's eye to drastically reduce light reflection.
This nanoporosity causes a gradual change in silicon density with depth, with a related change in its refractive index-a measure of how much the velocity of a light wave is reduced within the material. The important result is that light entering the cell does not encounter any sharp interfaces that would reflect the ray out of the cell and waste energy that could have produced electricity.

The well-known "frog in a kettle" story provides a simple analogy to the response of the incoming light to the nanoporosity created by NREL's black silicon etch. The frog starts out in a kettle of cold water, but does not perceive that the water is gradually being heated until it begins to boil and it's too late. In the happier black silicon story, light starts out travelling through low-refractive-index air, but does not "perceive" that the index is gradually increasing until it finally reaches that of the non-porous silicon at depth. Just as the frog would have jumped out of the kettle if it had felt the water getting too hot, so also the light would have been reflected out of the wafer if it had encountered a significant contrast in refractive index. The change in index with depth was so small as to be imperceptible to the light-yet the light went from one medium to another with a much different property without detecting an interface and being reflected out of the silicon.

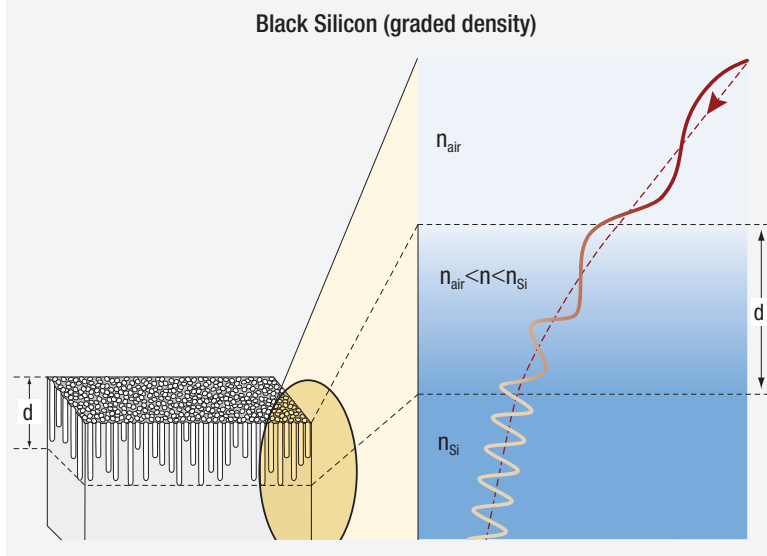

Schematic of a graded-density surface zone of thickness "d" that suppresses light reflection. The expansion shows that the wavelength of light in the non-porous silicon at depth is about one-third that in air. The graded zone shows the transition of wavelength between these two extremes without any reflection because of the gradual change in density and refractive index (" $n$ ") with depth. The wafer appears black because all light is being absorbed. 


\section{Benefits Galore}

The cumulative impact of NREL's highly lightabsorbing black silicon includes the following:

- Reduced module cost. NREL's etch also provides a direct reduction of module cost of $\$ 0.02$ to $\$ 0.04$ per watt of delivered module power-or $\$ 4$ to $\$ 8$ off the cost of a typical 200-watt solar panel.

- Reduced capital costs and better environmental safety. Assuming equivalent cell efficiencies, NREL's black silicon reduces capital costs by $16 \%$, while reducing operating expenses and eliminating environmentally harmful cleaning gases and dangerous silane gas. This capital cost reduction reduces the barrier to PV plant expansion and could speed the deployment of PV.

- Improved cell efficiency. Increases in manufactured cell efficiency of up to $0.8 \%$ absolute are possible compared to conventional industry techniques. This improvement can reduce silicon PV module costs by \$20 per panel.

- Reduced balance-of-system costs. Increased efficiency and energy production of PV modules reduce the balance-of-system (BOS) costs that are area-related: if the required module area is reduced due to higher efficiencies, the BOS costs are likewise reduced.

- Versatile range in incident light angle. The superior angle-of-incidence performance of black silicon antireflection may result in $1 \%$ to $3 \%$ more energy produced per rated-module kilowatt because of better morning, evening, and diffuse light performance.

Taken together, these improvements resulting from NREL's black silicon process could reduce the levelized cost of energy from a silicon PV array by about $2.5 \%$.

\section{Moving into the Market}

Two companies are negotiating licenses to NREL's black silicon patents. One company is pursuing broad use of the technology by the PV industry as a cost-effective replacement for silicon solar cell antireflection coatings; the other company will take advantage of another feature of black silicon to improve a proprietary system for collecting solar energy.

The ultimate market for the black silicon technology will be the manufacturers of PV solar cells, under sublicense from the equipment and etchant suppliers. PV manufacturers purchased more than $\$ 4$ billion in equipment in 2008 , and they seek every possible cost and efficiency advantage over their competitors.

\section{Clear Advantages of NREL's Black Silicon}

The innovation of NREL's black silicon approach garnered a 2010 R\&D 100 Award. The NREL technology tallied clear benefits compared to its two competitor fields: the current standard industry antireflection treatments using plasmaenhanced chemical vapor deposition or physical vapor deposition, and other black silicon treatments developed by a variety of other companies or university research groups.

Fabrication costs of techniques developed by others to create black silicon nanoporosity have been high, and the efficiencies of cells without additional antireflection coatings have not exceeded $13.9 \%$. NREL has validated cells using its black silicon alone at $16.8 \%$ conversion efficiency.
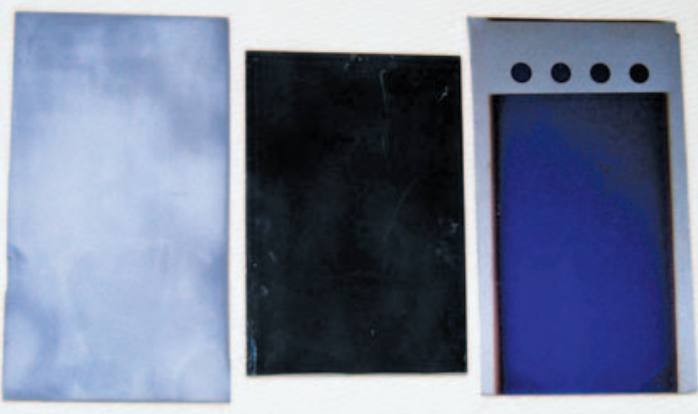

Photo of silicon wafers with antireflection treatments: (Left) with industrial micron-scale texture; (Right) with micron-scale texture plus an interference coating; and (Center) after NREL's single-step liquid etch. Note that the NREL cell is black because it absorbs almost all incident light.

\section{For More Information}

Technical: Howard Branz 303-384-6694

howard.branz@nrel.gov 